\title{
Modelos educativos y perfiles de los docentes de Bibliotecología y Ciencia de la Información en Venezuela
}

\section{Educational Models and Profiles of Teachers of Library Science and Information Science in Venezuela}

\author{
Johann Pirela Morillo * \\ Universidad de La Salle, Colombia
}

\section{Resumen}

En este artículo se presentan resultados de una investigación que tuvo como objetivo analizar los modelos educativos predominantes en las Escuelas y Programas de Bibliotecología y Ciencia de la Información, de instituciones de educación superior venezolanas y los perfiles de los docentes que se desempeñan en tales escuelas y programas. Los referentes conceptuales incluyeron una comparación de modelos para estructurar perfiles de los académicos con base en el enfoque por competencias, por ser el de mayor incorporación en el currículo para formar profesionales de Bibliotecología y Ciencia de la información en Venezuela.

Algunos resultados reportan que el mayor porcentaje en edad de los docentes encuestados se ubica en el rango entre 45 a más de 51 años, notándose un equilibrio en cuanto al género, aspecto que ha venido variando en los últimos años, porque la docencia bibliotecológica en el país era mayormente ejercida por mujeres. En cuanto a la formación académica, el mayor porcentaje se ubicó en el área de Bibliotecología y Ciencia de la Información, pero es interesante observar la tendencia hacia la interdisciplinariedad, sobre la base de considerar áreas como: Comunicación Social, Letras (Lingüística y Literatura) e Ingeniería. Las conclusiones señalan que existe pertinencia entre los modelos de las instituciones y los perfiles de los docentes, quienes evidenciaron interés en 
cuanto a la formación y actualización pedagógica y didáctica, como una estrategia que se revierte en ventajas para la formación profesional en el área.

Palabras clave: Modelos educativos, perfiles docentes, enseñanza de la Bibliotecología y Ciencia de la Información, perfiles docentes por competencias.

\section{Abstract}

The results of an investigation that had as objective to analyze the predominant educational models in the Schools and Programs of Librarianship and Information Science, of Venezuelan institutions of higher education and the profiles of the teachers who work in such Schools and Programs. Conceptual references included a comparison of models to structure profiles of academics based on the competency approach, since it is the one with the highest incorporation in the curriculum to train professionals in Librarianship and Information Science in Venezuela.

Some results report that the highest percentage of teachers surveyed age is in the range of 45 to over 51 years, with a gender balance. This aspect has been changing in recent years, because library teaching in the Country was mostly exercised by women. As for academic training, the highest percentage was in the area of Library and Information Science, but it is interesting to observe the trend towards interdisciplinarity, based on considering areas such as: Social Communication, Literature (Linguistics and Literature) And Engineering. The conclusions indicate that there is relevance between the models of the institutions and the profiles of teachers, who showed interest in the training and pedagogical and didactic updating, as a strategy that reverts to advantages for professional training in the area.

Key words: Educational models, teaching profiles, Library and Information Science teaching, teaching profiles by competences. 


\section{Introducción}

Se parte de la premisa de que los modelos educativos son el conjunto de concepciones, paradigmas y sistemas teóricos que fundamentan los procesos de aprendizaje en las instituciones educativas. En el contexto de la enseñanza superior constituyen parte importante del nivel macro-curricular, en el cual se expresa el ideal de persona-profesional que se pretende formar, articulada a su vez con una utopía social que se pretende construir. En este trabajo se analiza la pertinencia de los modelos educativos de las instituciones de educación superior que en Venezuela tienen el compromiso de formar los profesionales en Bibliotecología y Ciencia de la Información y los perfiles de los docentes, entendiendo por estos los conocimientos, habilidades, destrezas y actitudes que los maestros debemos exhibir, los cuales deben estar alineados con los principios establecidos en los modelos para convertirlos en una realidad concreta.

Se ha mencionado repetidas veces que el éxito de la ejecución del currículo de toda institución educativa, depende en gran medida de la preparación profesional, pedagógica y académica de los docentes, en tanto que actores esenciales del proceso formativo. Esta idea se rescata en este trabajo para enfatizar la importancia que se debe apreciar entre los modelos educativos, los perfiles de las instituciones de educación superior y los perfiles de los docentes universitarios, quienes tienen en sus manos una gran responsabilidad, relacionada con la preparación de los cuadros profesionales que se requieren para apalancar procesos de desarrollo.

En este sentido, se analiza la pertinencia que existe entre los modelos educativos expresados en las universidades venezolanas formadoras de profesionales de Bibliotecología y Archivística y los perfiles de los docentes. Cabe mencionar que este artículo recoge los datos sobre Venezuela del Proyecto internacional e interinstitucional titulado: Perfil del docente y su vinculación con el modelo educativo en la educación bibliotecológica y de documentación Iberoamérica, coordinado por la Dra. Lina Escalona Ríos del Instituto de Investigaciones Bibliotecológicas y de la Información, de la Universidad Nacional Autónoma de México (IIBI- 
UNAM) y en el cual participan investigadores de México, Venezuela, Colombia, Costa Rica y España.

\section{Objetivo}

Analizar la pertinencia entre los modelos educativos predominantes en las Escuelas y Programas de Bibliotecología y Ciencia de la Información de instituciones de educación superior venezolanas y los perfiles de los docentes.

\section{Desarrollo}

Los modelos educativos se han venido configurando a partir de las condiciones sociohistóricas, culturales y políticas que dieron origen a variadas formas de concebir la educación, sus procesos, instituciones y actores fundamentales. En cada modelo subyace una idea de docente, de estudiante y un sistema de relaciones y significados bajo los cuales se entiende la enseñanza y el aprendizaje. En este sentido, desde los modelos conductistas hasta los más actuales, centrados en el constructivismo y las competencias, se ha propugnado por el hecho de que cada modelo encarna también una concepción del currículo y el conjunto de conocimientos, habilidades, destrezas y actitudes requeridas en los profesionales de la docencia.

Los modelos construidos para la enseñanza de la Bibliotecología y la Ciencia de la Información no escapan de estas dinámicas, en virtud de que han estado incorporando las lógicas sobre el proceso formativo, atendiendo a elementos dados por la sociedad y cultura donde tienen sentido. Los modelos educativos, entonces, se traducen en función de un sistema de representación de variables e indicadores que definen la estructura socio-cultural, que reconoce el valor de la formación profesional para alcanzar mayores niveles de desarrollo. En este sentido, se entienden como una representación de la naturaleza y alcance acerca del desempeño que deben tener los actores del proceso educativo. 
Es así como en el modelo conductista, la idea que se tiene del docente es un transmisor de información y contenidos que los estudiantes deben demostrar en pruebas orientadas hacia la comprobación del grado de dominio de tales contenidos. En los modelos centrados en las pedagogías críticas, humanistas, constructivistas y finalmente en el enfoque por competencias, se le pretende dar un mayor protagonismo a los estudiantes, asumiéndolos como gestores activos de sus propios procesos de aprendizajes, los cuales no se conciben como logros finales y acabados, sino como estadios previos desde donde será posible ascender hacia otros niveles de interacción y construcción de conocimientos, siempre perfectibles y en una perspectiva de mejoramiento continuo, que conllevan a prolongar y visualizar de forma permanente zonas de desarrollo próximo, cada vez más exigentes y retadoras.

Estos modelos apuestan por una idea de docente como mediador de experiencias significativas de aprendizajes, como un traductor y organizador de símbolos a los cuales habrá que imprimirles sentido, por medio de la activación de procesos de pensamiento. Esta perspectiva alude también al carácter estratégico que tiene la intencionalidad y trascendencia de los procesos de aprendizaje, lo cual además requerirá de planear las acciones didácticas de forma consecuente con los principios señalados en los modelos.

Se debe observar una relación de correspondencia entre los modelos educativos, los perfiles institucionales y los perfiles de los docentes de Bibliotecología y Ciencia de la Información, con lo cual se estará en mejores condiciones para conseguir la pertinencia de la formación profesional en este campo, signado por importantes mutaciones que implican el tránsito hacia nuevos modos de concebir la realidad y de participar en ella. La Figura 1 ilustra la correspondencia entre el modelo educativo, el perfil institucional y el perfil del docente.

La adopción de uno u otro modelo o de opciones teóricas y metodológicas eclécticas, requieren de nuevos docentes comprometidos con la formación pedagógica, curricular y didáctica, por cuanto no basta con dominar conocimientos disciplinares o prácticos propios del campo de conocimiento de la Bibliotecología y Ciencia de la Información, sino que es preciso 
construir saberes sobre el ser, hacer y conocer de la enseñanza y aprendizaje de la disciplina y su práctica profesional.

De este modo, los modelos teóricos orientadores de la formación en el campo deben visualizarse en relación con los perfiles de las instituciones educativas y con los propios perfiles de los docentes, configurados en torno a las trayectorias pedagógicas y didácticas, a las experiencias de diseño, construcción y evaluación curricular y otras interacciones mediadas por los procesos de investigación, desde donde se generan importantes insumos que enriquecen las prácticas pedagógicas que fomentan una formación profesional competitiva y consustanciada con los cambios y retos impuestos por las sociedades de la información y el conocimiento.

La figura No. 1 ilustra la relación de implicación que se debe observar entre los modelos educativos, los perfiles instituciones y los perfiles de los docentes para que la ejecución curricular sea exitosa y comprometida.

Figura 1

Relación entre el modelo educativo y el perfil de los docentes

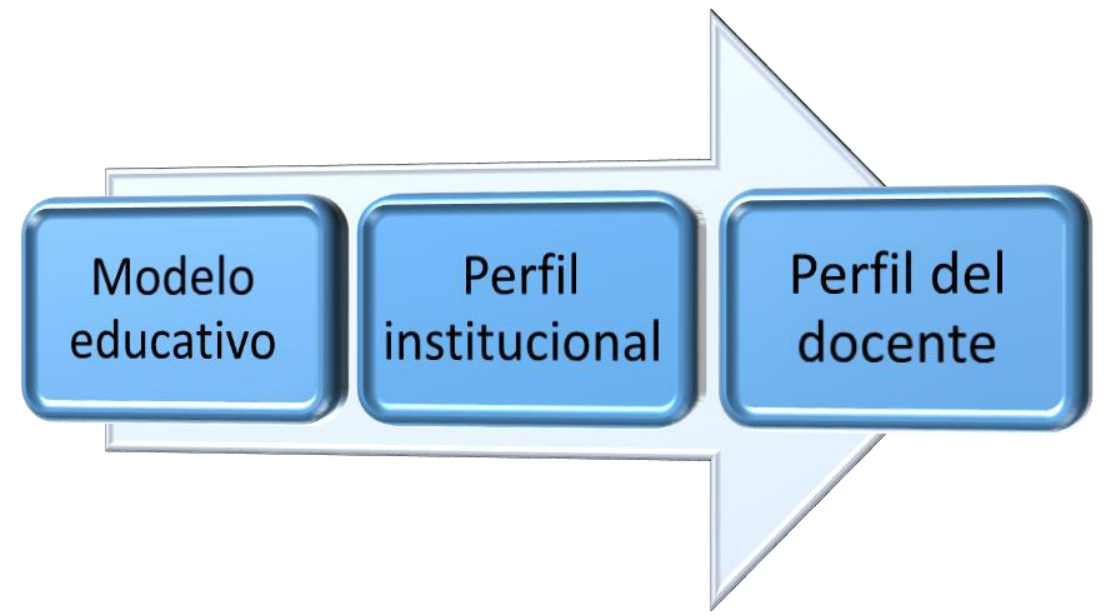

Fuente: elaboración propia del investigador (2017) 
En cuanto a los modelos educativos de las universidades venezolanas que ofrecen Escuelas y Programas de Bibliotecología y Ciencia de la información, se tiene que estos se enfocan desde modelos centrados en la integralidad, el aprender haciendo y las competencias. Estos modelos hacen pensar en perfiles de docentes en los cuales se pongan de relieve nuevos saberes y prácticas que trasciendan la sola transmisión de información o de contenidos, para dar cabida a las nuevas racionalidades de los procesos de mediación del aprendizaje que convocan acciones mucho más intencionadas, creativas e interdiscursivas.

Frente a la caracterización de los modelos educativos también se ha estado planteando un conjunto de propuestas para perfilar las competencias que los docentes deben exhibir y con ello mostrarse en sintonía con las realidades complejas, interactivas y globales que imponen las denominadas sociedades de la información y del conocimiento, para cuya construcción efectiva, desde la educación, se requerirá trascender la transmisión de contenidos, abriendo paso a formas mucho más dinámicas y flexibles, que le otorguen especial prevalencia al aprender con sentido y significado, con base en el desarrollo de competencias en el uso estratégico de la información.

Algunas de las propuestas para establecer competencias que deben desarrollar los docentes, en atención a los nuevos modelos educativos, son las de Tobón y Tobón (2010), la Consejería de Castilla y León (2011), Álvarez (2011), la Universidad del Zulia de Venezuela (2012) y Guzmán, Marín e Inciarte (2014). Todas estas propuestas coinciden en plantear que los docentes actualmente deben desarrollar conocimientos, habilidades, destrezas y actitudes para la gerencia académica, la comunicación didáctica, mediada por tecnologías digitales, el fomento de la metacognición y autogestión del propio aprendizaje y la investigación como una acción que debe permear los procesos formativos.

De las propuestas señaladas las más actuales y pertinentes con los modelos educativos de las instituciones venezolanas formadoras de profesionales de Bibliotecología y Ciencia de la Información son las de Tobón y Tobón (2010) y la de Guzmán, Marín e Inciarte (2014). De acuerdo con lo planteado por Tobón (2010), las acciones y competencias que todo docente 
debe desarrollar en el actual contexto social son:

- Acción 1: Direccionar y ordenar su rol docente en base a metas claras y pertinentes.

- Acción 2: Planear, con flexibilidad, las actividades a realizar.

- Acción 3: Actuar con los estudiantes, buscando que estos logren el desarrollo de competencias.

- Acción 4: Evaluar lo realizado mediante el mejoramiento continuo.

Sobre las competencias, el mencionado investigador colombiano señala que estas deben apuntar a:

- Trabajo en equipo

- Comunicación

- Planeación del proceso educativo

- Evaluación del aprendizaje

- Mediación en el aprendizaje

- Gestión curricular

- Producción de materiales

- Tecnologías de la información y la comunicación

- Gestión de la calidad del Aprendizaje

Asimismo, Tobón (2010) propone que deben existir otras acciones claves para el perfeccionamiento docente, entre las que describe:

- Acción 1: Observar y analizar ejemplos de buenas prácticas docentes para trascender el aprendizaje y las experiencias percibidas en los libros y entrar en contacto con estrategias y aspectos específicos de situaciones, estudiantes y contextos particulares.

- Acción 2: Autoevaluarse en función de retos y logros en el ejercicio de la docencia, teniendo el valor de asumir la mejora continua como una acción permanente.

- Acción 3: Poner en acción las competencias docentes por medio de acciones puntuales en clases y tomando en cuenta las competencias que deben desarrollar 
los estudiantes, así como la flexibilidad necesaria en el proceso de mediación de los aprendizajes.

- Acción 4: Evaluación y certificación externa de las competencias docentes presentando evidencias de su puesta en acción con los estudiantes, ante otros docentes, instituciones y organizaciones con el fin de tener retroalimentación sobre el nivel alcanzado de dichas competencias y lograr un grado de evaluación que permita, además de certificar dichas competencias, encontrar aspectos 0 consideraciones a mejorar.

Por otra parte, estas competencias pueden ser ampliadas con lo expuesto por Guzmán, Marín e Inciarte (2014) quienes establecen un sistema de 7 competencias para evaluar el desempeño académico del docente. Al respecto señalan los siguientes criterios:

- Desarrolla su formación continua: gestiona el proceso de análisis, maduración y desempeño de sus competencias docentes visualizando la brecha entre el ideal y su ejercicio cotidiano y estableciendo metas personales y profesionales para su superación.

- Realiza procesos de trasposición didáctica: reconoce, se involucra y desempeña de forma consciente la transformación de los contenidos para lograr transmitirlos al estudiante de manera efectiva.

- Diseña su docencia mediante dispositivos de formación y evaluación de competencias: el docente parte de los contenidos y propósitos formativos de su materia para desarrollar métodos, medios, materiales, procesos y estrategias que permitan el desarrollo y evaluación de competencias en sus estudiantes.

- Gestiona la progresión de la adquisición de competencias: Considerando los itinerarios de formación y la evolución de los niveles de desempeño de los estudiantes mientras observa y evalúa las situaciones de aprendizaje con la aplicación de controles y aplicando además controles y correcciones periódicas para garantizar el cumplimiento de las metas de cada fase del proceso pedagógico.

- Coordina la interacción pedagógica: practicando una docencia basada en situaciones que se ejerce mediante la puesta en práctica de dispositivos, procesos y estrategias 
en torno al logro de las competencias, buscando una interacción sustentada en la comunicación horizontal que genere ambientes de aprendizaje significativo y redes de colaboración.

- Aplica formas de comunicación educativa adecuadas: esta involucra tres microcompetencias íntimamente ligadas: la competencia en tecnologías de información y comunicación, el manejo de la información y la competencia en medios; mediante la articulación de las tres se perfila un individuo capaz de ver las TIC como recursos de apoyo en los procesos de aprendizaje que promueve en sus estudiantes la búsqueda, procesamiento e interpretación de información.

- Valora el logro de competencias: aplicando dispositivos, estrategias e instrumentos que le permitan evaluar el desempeño de sus estudiantes en el logro de las competencias establecidas para su asignatura, valorando, además, el impacto personal de la experiencia didáctica en el alumno.

\section{Metodología}

La metodología fue de enfoque cuantitativo, sustentada en la técnica de la encuesta que se desarrolló a partir de un cuestionario estructurado, diseñado por el equipo integrante del Seminario Internacional de Educación Bibliotecológica, coordinado por la Dra. Lina Escalona Ríos de la Universidad Nacional Autónoma de México. El cuestionario abordó indicadores relacionados con el perfil profesional de los docentes, con objeto de determinar si dicho perfil se encuentra en sintonía con los modelos educativos predominantes en las Escuelas y Programas de Bibliotecología y Ciencia de la Información de Venezuela.

Cabe destacar que el instrumento se elaboró para ser enviado y respondido en línea. En el caso de Venezuela respondieron el instrumento 25 profesores, distribuidos entre todas las instituciones de educación Superior que ofrecen programas académicos en el área. De este total de respuestas, el $44 \%$ corresponde a docentes de la Escuela de Bibliotecología y Archivología de la Universidad Central de Venezuela, el 20\% a docentes de la Escuela de Bibliotecología y Archivología de la Universidad del Zulia, el 32\% a la Universidad Politécnica 
Territorial de Lara "Andrés Eloy Blanco" y el 4\% restante a la Universidad de Yacambú, tal y como se muestra en el Gráfico 1.

\section{Gráfico 1}

\section{Distribución de docentes por Universidad}

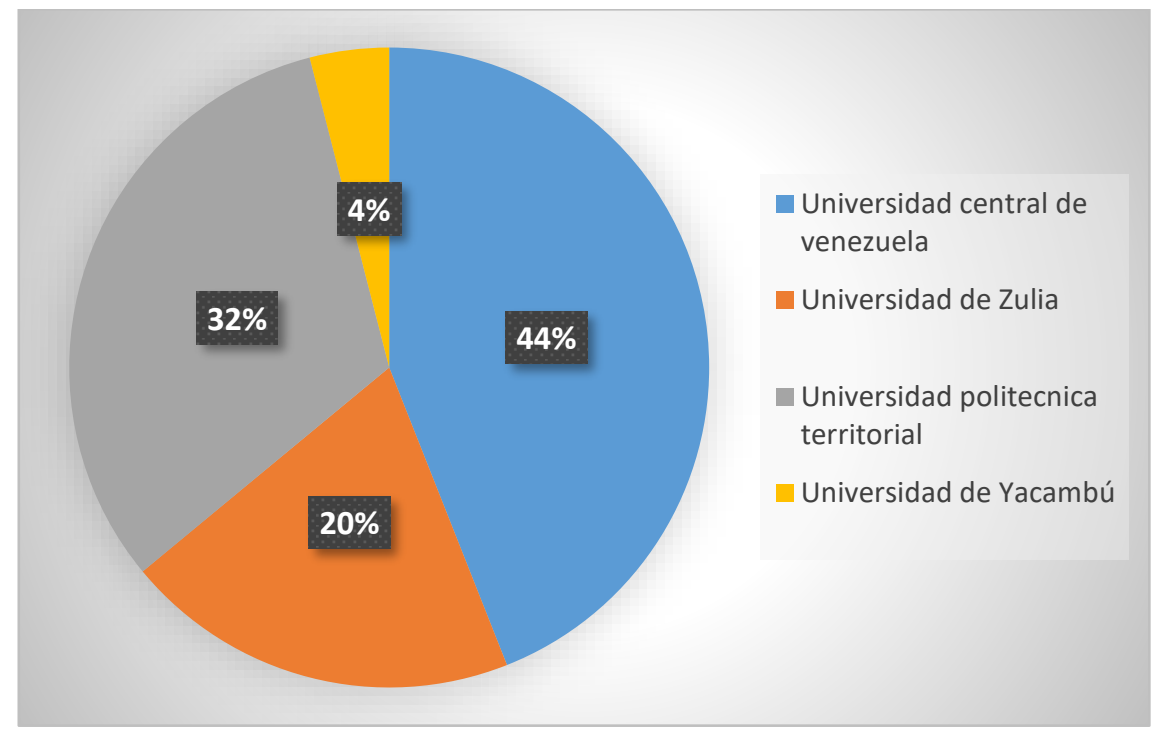

Fuente: elaboración propia del investigador (2017)

\section{Discusión de resultados}

En cuanto a los indicadores: género, edades y niveles donde se desarrolla la docencia, se encontró que los docentes encuestados en su mayor parte son mujeres (56\%) y el $44 \%$ restante son hombres. Las edades de mayor recurrencia son las de más de 46 años (68\%), entre 36 a 45 años (24\%) y entre 25 y 35 años (8\%). Finalmente, en cuanto a los niveles educativos donde se desarrolla la docencia, se evidenció que el postgrado con un $52 \%$ y el pregrado con el $42 \%$, otro nivel (que puede ser el de técnico) se reportó con un $6 \%$.

A partir de estos datos se puede comentar el desplazamiento que se va observando en el país, en relación con el balance entre la población masculina y femenina, en virtud de que habitualmente los docentes del área de Bibliotecología y Ciencia de la Información habían sido mayormente mujeres. Sobre las edades, también se aprecia un dato interesante, el cual indica la madurez cronológica y académica de los docentes venezolanos dedicados a la 
enseñanza de la Bibliotecología, notándose una presencia baja de docentes que van de los 25 a los 35 años. Igualmente, se observa la presencia de docentes del área como profesores de postgrado. En síntesis, el docente venezolano de Bibliotecología es una mujer, entre edad de madurez cronológica y académica que ejerce la docencia tanto en pregrado como en postgrado (Ver Gráficos 2, 3 y 4).

\section{Gráfico 2}

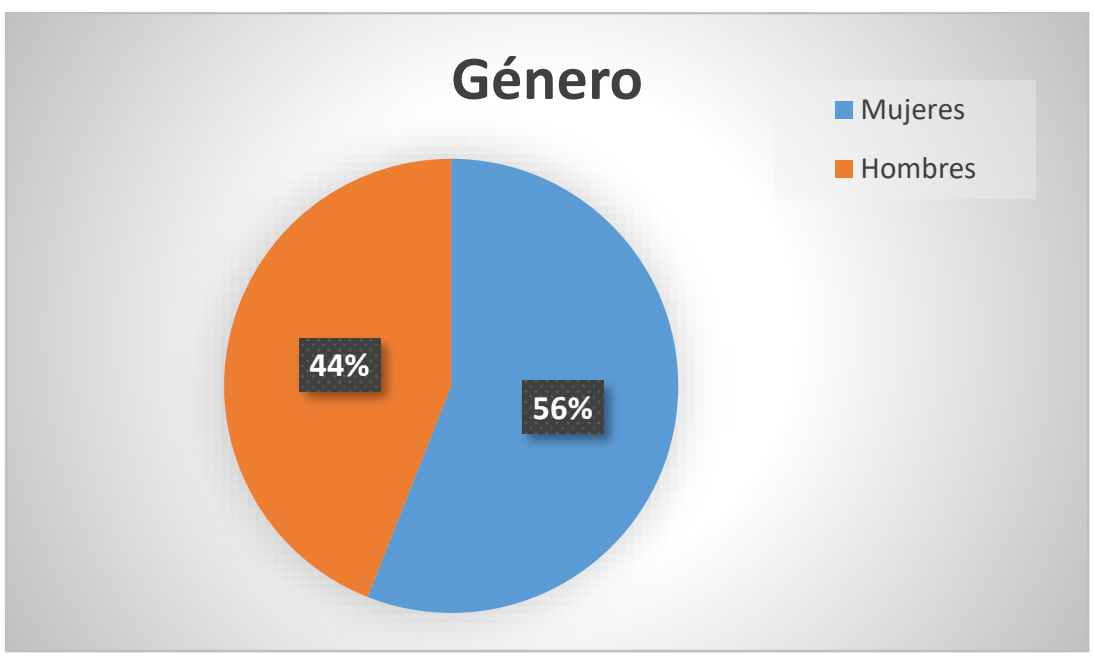

Fuente: elaboración propia del investigador (2017)

\section{Gráfico 3}

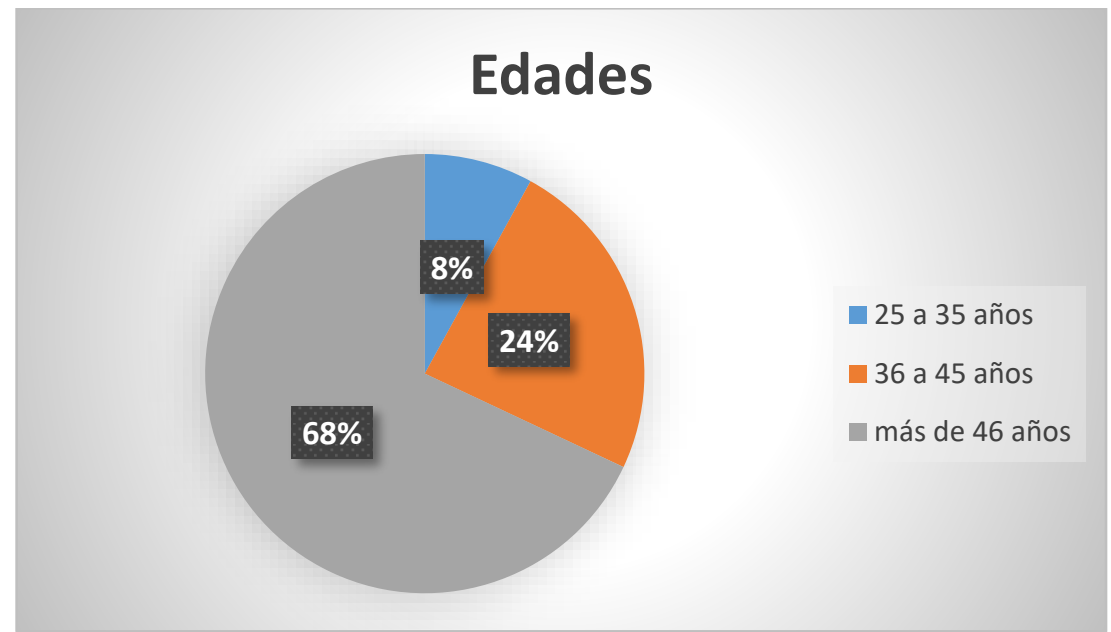

Fuente: elaboración propia del investigador (2017) 


\section{Gráfico 4}

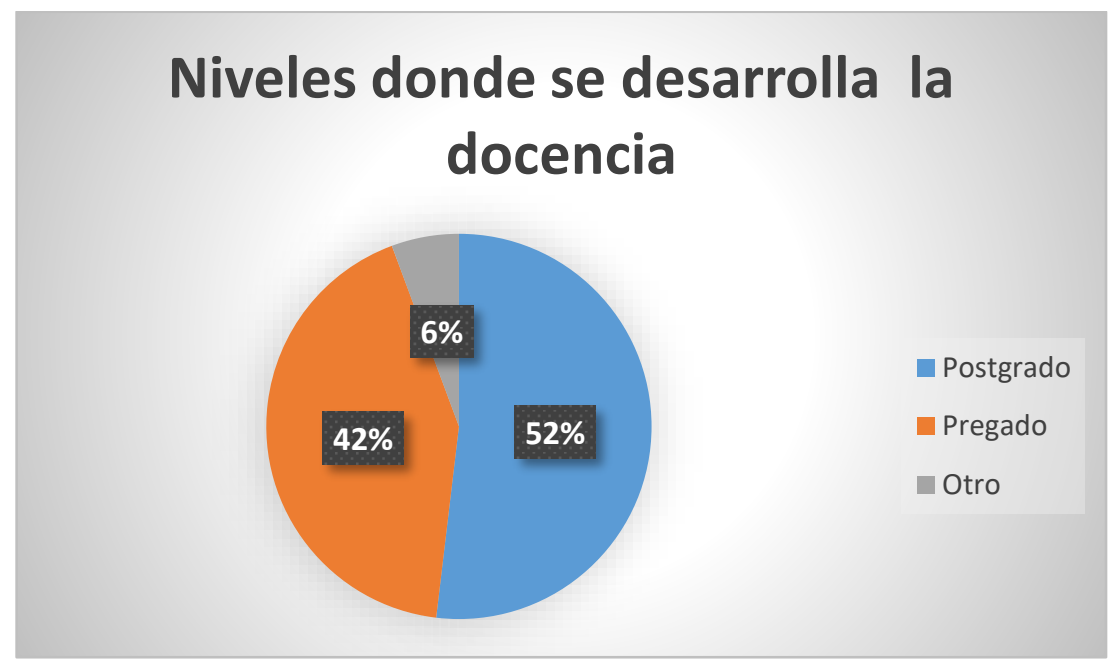

Fuente: elaboración propia del investigador (2017)

Sobre los indicadores, relacionados con la situación de dedicación actual de los profesores y la formación: académica (de pregrado), disciplinar y la actualización permanente en postgrado, se reportó un alto porcentaje de tiempo parcial (49\%), frente a un $44 \%$ de tiempo completo y el $7 \%$ otro tipo de dedicación, básicamente de tiempo ocasional o convencional. La formación académica de pregrado de los docentes evidenció un alto porcentaje (75\%) de formación en el área de Bibliotecología y Ciencia de la Información, con el restante $25 \%$ repartidas en áreas como: Comunicación Social, Letras (Lingüística y Literatura) e Ingenierías.

En cuanto a la formación de postgrado, el $45 \%$ se concentró en el área de gerencia, distribuida entre educativa y empresarial; el 38\% tiene formación de postgrado en el área de Bibliotecología y Ciencia de la Información y el $8 \%$ en el área de las ciencias de la comunicación. Al correlacionar estos tres indicadores se observan aspectos de singular importancia. El primero es que existe un alto porcentaje de docentes de tiempo parcial, lo cual podría obstaculizar el desarrollo y ejecución efectiva de proyectos curriculares institucionales, para cuya concreción efectiva se requiere que los docentes dediquen un tiempo prolongado, con lo cual también se compromete el sentido de pertenencia y el grado de implicación o involucramiento con las instituciones. 
El segundo de los aspectos que saltan a la vista es la tímida incorporación que se aprecia en las Escuelas y Programas de docentes provenientes de otras áreas distintas a la Bibliotecología y la Ciencia de la Información, lo cual favorece la interdisciplinariedad no solo de la planta docente, sino el dinamismo que se le imprime a la formación académica de los profesionales del área. El tercero de los elementos que consideramos relevante es la orientación y perfiles de formación de postgrado, los cuales se mueven entre las ciencias gerenciales, la propia Bibliotecología y Ciencia de la Información y las ciencias de la comunicación, esta última sobre todo se observa con mucha fuerza en la Escuela de Bibliotecología y Archivología de la Universidad del Zulia, cuyos docentes cuentan con formación de maestría en este campo, abarcando desde las nuevas tecnologías de información y comunicación, la socio-semiótica de la comunicación y la cultura y la gerencia de la comunicación (Ver Gráficos 5, 6 y 7).

\section{Gráfico 5}

\section{Situación actual como profesor}

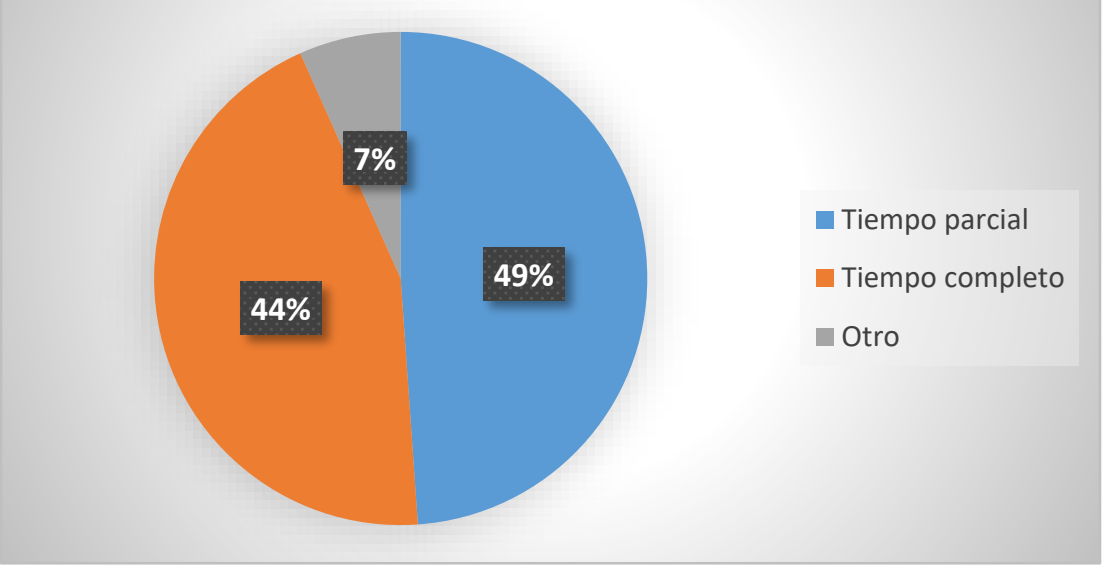

Fuente: elaboración propia del investigador (2017) 


\section{Gráfico 6}

\section{Formación académica -} pregrado

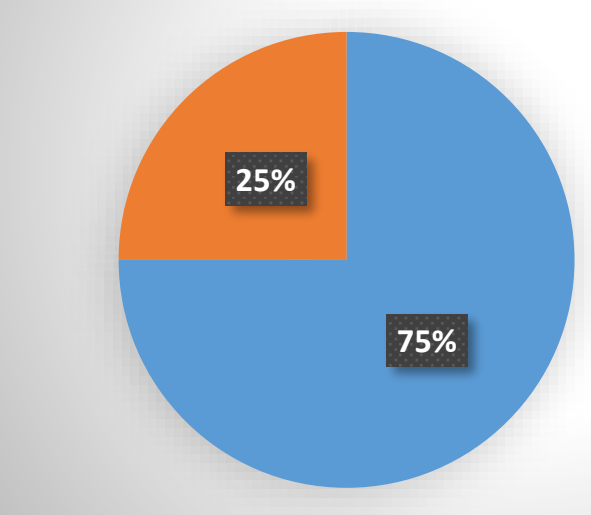

- Licenciatura en Bibliotecología y Archivología

- Letras, comunicación social e Ingenierías

Fuente: elaboración propia del investigador (2017)

\section{Gráfico 7}

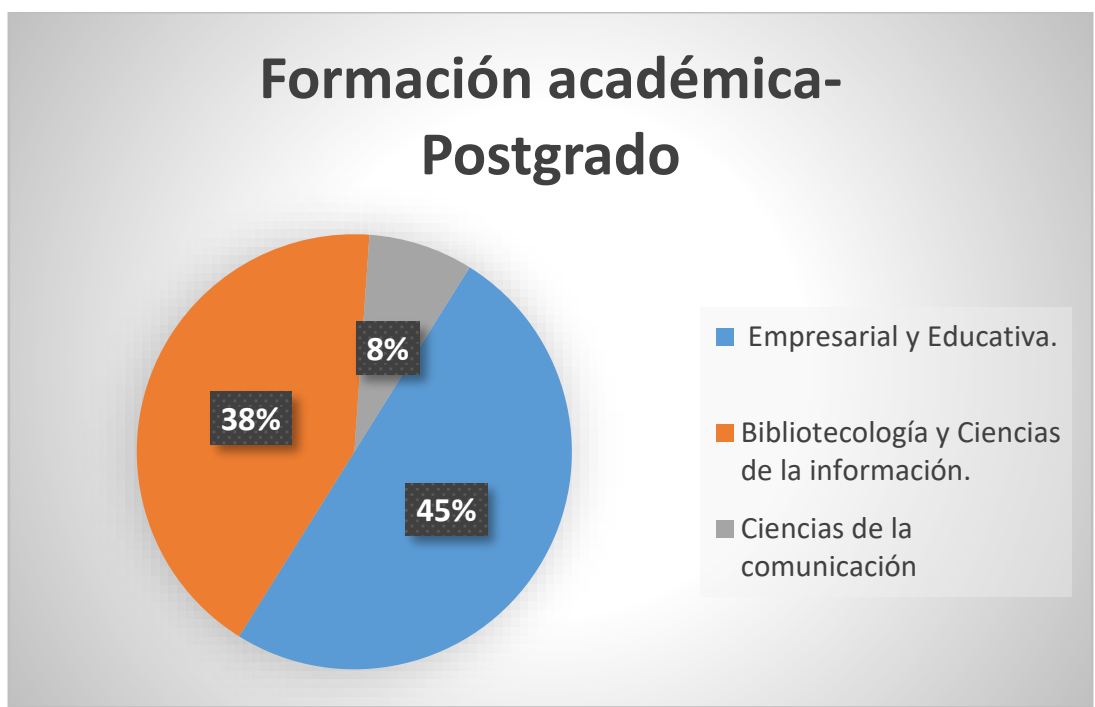

Fuente: elaboración propia del investigador (2017)

Finalmente, en cuanto a los cursos disciplinares de actualización y perfeccionamiento, la formación pedagógica y la experiencia laboral tenemos que el $46 \%$ ha tomado cursos de actualización en los últimos tres años, el $53 \%$ ha tomado cursos de formación pedagógica y el $45 \%$ ha acumulado una experiencia laboral que va de los 21 a los 40 años. Estas 
frecuencias reportadas con mayores puntajes permiten señalar que existe un alto porcentaje que no ha tomado cursos de actualización y perfeccionamiento en el área específica de la disciplina bibliotecológica y de la ciencia de la información, lo cual llama la atención, en virtud de los últimos cambios registrados, sobre todo en cuanto a la integración en la gestión digital de contenidos, el análisis y descripción bibliográfica y documental, con la introducción y uso de los nuevos estándares para el análisis como las RDA, por ejemplo.

También se rescata como dato importante el porcentaje importante de docentes que han realizado cursos y diplomados para apropiarse de competencias docentes, de lo cual depende que se logre articular una formación para enfrentar con creatividad y alto nivel de sensibilidad los procesos educativos y evaluativos en las instituciones de educación superior. En relación con la experiencia en el ejercicio profesional, se reportó un número importante de docentes con más de 20 años de experiencia frente a un porcentaje mayor (55\%) con experiencia entre 1 y 20 años. (Ver Gráficos 8, 9 y 10).

\section{Gráfico 8}

\section{En los tres últimos años cursos disciplinares}

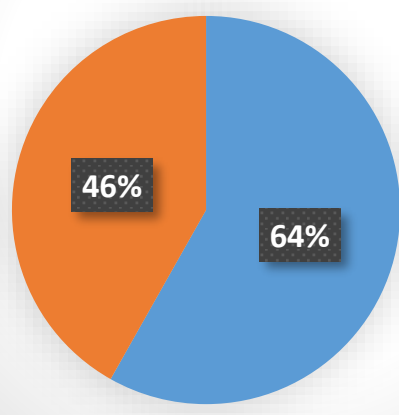

Fuente: elaboración propia del investigador (2017) 


\section{Gráfico 9}

\section{Cursos de formación pedagógica}

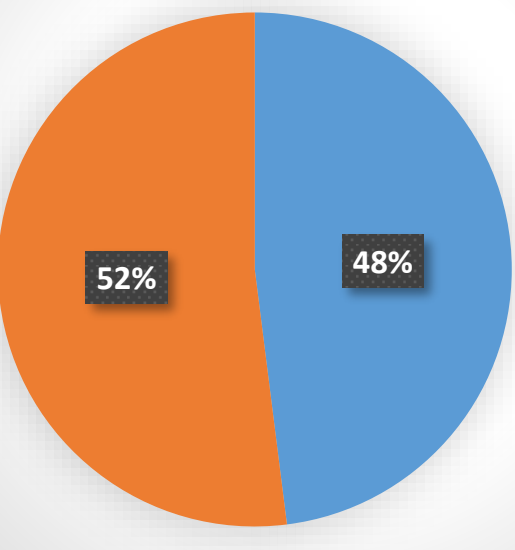

$$
\square \mathrm{NO} \square \mathrm{SI}
$$

Fuente: elaboración propia del investigador (2017)

\section{Gráfico 10}

\section{Experiencia laboral}

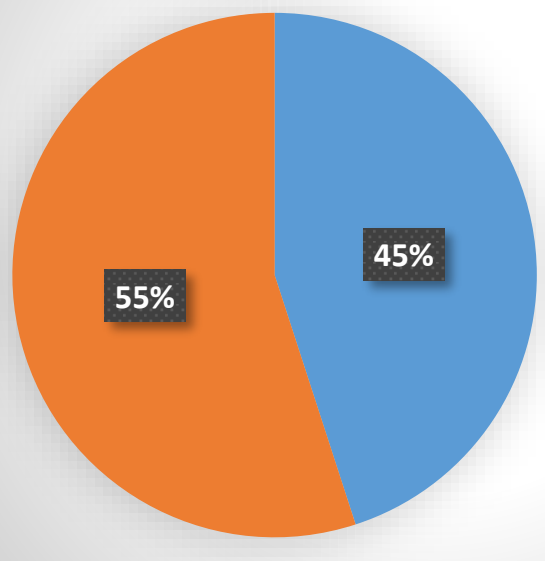

Entre 21 a 40 años.

Fuente: elaboración propia del investigador (2017) 


\section{Conclusiones}

El análisis realizado sobre la relación entre los modelos educativos relacionados con los perfiles de los docentes de las Escuelas y Programas de Bibliotecología y Ciencia de la información permite enfatizar en la necesidad de establecer las condiciones para acercar cada vez las aspiraciones educativas expresadas en tales modelos con los perfiles en los que se delinean los conocimientos, habilidades, destrezas y actitudes de los docentes, quienes deben desarrollar acciones intencionadas y articuladas con los propósitos formativos.

Este estudio evidenció que los perfiles de los docentes de Bibliotecología y Ciencia de la información en Venezuela expresan una alta madurez académica, dato que correlaciona también con la experiencia importante acumulada en la práctica profesional del campo de la Bibliotecología y Ciencia de la información. Igualmente, se reveló el interesante tránsito que se está dando hacia lo interdisciplinario, elemento que empodera a las Escuelas y Programas hacia el desarrollo de apuestas formativas que superan la visión tradicional y parcelada del conocimiento generado y socializado en la docencia. Se considera que este carácter interdisciplinario se debe seguir intensificando para garantizar que los futuros profesionales cuenten con herramientas para el trabajo en equipo y las interacciones necesarias para ampliar los horizontes de sentido del campo bibliotecológico e informacional.

En cuanto a la formación de postgrado, el perfil docente está concentrado hacia las áreas de gerencia y las ciencias de la comunicación, con un porcentaje importante también hacia el propio bibliotecológico y de ciencia de la información. La formación pedagógica es un elemento que aparece en muchos perfiles docentes, pero se considera que hay que seguir profundizando en esta, hasta el punto de configurar saberes pedagógicos y didácticos que orienten los procesos formativos en el área. 


\section{Referencias bibliográficas}

Álvarez, M. (2011). Perfil del docente en el enfoque por competencias. En: Revista Electrónica Educare Vol. XV, N 1, ISSN: 14094258, Enero-Junio, 2011. Recuperado https://dialnet.unirioja.es/descarga/articulo/3683582.pdf

Consejería de Castilla y León (2011). Modelo de competencias profesionales del profesorado.

Recuperado de: http://csfp.centros.educa.jcyl.es/sitio/upload/Modelo de Competencias Profesionales del Profesorado Definitivo JCyL.pdf

Guzmán, I., R. Marín y A. Inciarte. (2014). Innovar para transformar la docencia universitaria. Un modelo para la formación por competencias. Maracaibo: Astrodata. Universidad del Zulia.

Tobón, S. (2010). Proyectos Formativos: Metodología para el desarrollo y evaluación de las competencias. México DF. CIFE.

Universidad del Zulia. (2012). Diseño curricular de la Escuela de Educación. Facultad de Humanidades y Educación. Escuela de Educación. Maracaibo: Venezuela.

\section{Notas del Autor}

* Johann Pirela Morillo: Universidad de La Salle, Colombia. Correo electrónico: kikepirela@gmail.com 